\title{
Polydatin alleviates non-alcoholic fatty liver disease in rats by inhibiting the expression of TNF- $\alpha$ and SREBP-1c
}

\author{
JINGMING ZHANG $^{1^{*}}$, YINGYING TAN $^{1^{*}}$, FANRONG YAO $^{2}$ and QI ZHANG ${ }^{1}$ \\ ${ }^{1}$ Department of Traditional Chinese Medicine, Basic Medical College, Shaanxi University of Chinese Medicine, Xianyang, \\ Shaanxi 712046, P.R. China; ${ }^{2}$ Department of Pharmaceutical Sciences, North Dakota State University, Fargo, ND 58102, USA
}

Received March 9, 2012; Accepted July 19, 2012

DOI: $10.3892 / \mathrm{mmr} .2012 .1015$

\begin{abstract}
The pathophysiology of non-alcoholic fatty liver disease remains incompletely elucidated, and available treatments are not entirely satisfactory. Polydatin, a stilbenoid compound derived from the rhizome of Polygonum cuspidatum, has been recognised to possess hepatoprotective and anti-inflammatory activities. The purpose of the present study was to determine whether polydatin has a protective effect against hepatic steatosis induced by a high-fat diet (HFD) and to elucidate its underlying molecular mechanisms in rats. Male Sprague-Dawley rats were randomly divided into four groups, including normal control, HFD model and polydatin-treated groups with polydatin levels of 30 and $90 \mathrm{mg} / \mathrm{kg}$. Following the experimental period, plasma total cholesterol (TC), triglyceride (TG) and hepatic lipid concentrations were determined. To identify a possible mechanism, we examined the changes in liver tumor necrosis factor- $\alpha$ (TNF- $\alpha$ ), lipid peroxidation levels and sterol-regulatory element binding protein (SREBP-1c) mRNA and its target genes. Both 30 and $90 \mathrm{mg} / \mathrm{kg}$ polydatin treatment alleviated hepatic steatosis and reduced plasma and liver TG, TC and free fatty acid (FFA) concentration significantly in HFD rats. In addition, TNF- $\alpha$, and malondialdehyde and 4-hexanonenal levels were markedly suppressed by polydatin in the liver of HFD-fed rats. Polydatin also decreased the gene expression of SREBP-1c and its target genes involved in lipogenesis, including fatty acid synthase (FAS) and stearoly-CoA desaturase 1 (SCD1) in HFD-fed rats. These results suggest that the protective effects of polydatin against HFD-induced hepatic steatosis may be partly associated with reduced liver TNF- $\alpha$ expression, lipid peroxidation level and SREBP-1c-mediated lipogenesis.
\end{abstract}

Correspondence to: Dr Qi Zhang, Department of Traditional Chinese Medicine, Basic Medical College, Shaanxi University of Chinese Medicine, Century Avenue, Xianyang, Shaanxi 712046, P.R. China

E-mail: zhangndsu@yahoo.com

*Contributed equally

Key words: hepatic steatosis, polydatin, fatty liver, tumor necrosis factor- $\alpha$, sterol-regulatory element binding protein

\section{Introduction}

Non-alcoholic fatty liver disease (NAFLD) is the most common type of liver disease and is closely associated with obesity and metabolic syndrome. There is a wide spectrum of pathologies covered by NAFLD, ranging from simple accumulation of triglycerides (TGs) in hepatocytes to non-alcoholic steatohepatitis (NASH) and in certain patients, this is followed by the progression to fibrosis and cirrhosis (1-3). The 'two-hit' hypothesis has become an important theoretical framework in understanding the pathogenesis of liver damage in these patients (3-6). Although initial and subsequent mechanisms are not entirely distinct, the first hit mainly consists of TG and fatty acid accumulation in the liver. The second hit involves oxidative stress and inflammation of the liver. Both conditions can be induced by high energy diets, and the central role of lipid accumulation in the liver in the pathogenesis of NAFLD has been confirmed in clinical correlation studies and animal models (6). Thus, decreasing serum and hepatic lipid levels are crucial for the prevention of NAFLD.

Polydatin is a stilbenoid compound derived from the rhizome of Polygonum cuspidatum. This plant has been used clinically in the treatment of digestive disorders and ischemia/ reperfusion injury in traditional Chinese medicine (7). One of the main properties of polydatin is the hepatoprotective activity, reportedly inducing gallbladder contraction, preventing biliary cholesterol-stone formation and protecting against tetrachloromethane and aflatoxin B1 hepatotoxicity (8-11). It has also been demonstrated that polydatin suppresses the oxidative and inflammatory damage in ischemic stroke (12). In addition, it has been revealed that treatment with polydatin significantly reduced the serum levels of total cholesterol (TC) and TGs in hyperlipidemic hamsters and rabbits induced by a high-fat diet (HFD) $(13,14)$. Despite these hypolipidemic and antioxidant activities, the extent to which polydatin improves hepatic steatosis has not been well studied.

The aim of this study was to assess the effects of polydatin on preventing hepatic lipid accumulation in high-fat diet-induced NAFLD rats. To investigate the possible mechanism, liver proinflammatory cytokine tumor necrosis factor- $\alpha$ (TNF- $\alpha$ ) and lipid peroxidation levels were determined. The principal regulator of hepatic fatty acid biosynthesis, sterol-regulatory element binding protein (SREBP-1c) and its 
response genes, fatty acid synthase (FAS) and stearoly-CoA desaturase 1 (SCD1) were also determined.

\section{Materials and methods}

Animals and treatment. Male Sprague-Dawley (SD) rats weighing 160-180 g were purchased from the Experimental Animal Center of Xi'an Jiaotong University. Animals were housed at $24^{\circ} \mathrm{C}$ with a $12 \mathrm{~h}$ light-dark cycle with free access to water and food. Animal experiments were approved by the Institute of Animal Use and Care Committee of the Shaanxi University of Chinese Medicine.

Rats received either a regular rodent chow (normal diet: $62.3 \%$ carbohydrate, $12.5 \%$ fat, $24.3 \%$ protein calories) or a HFD (42.0\% fat, $36.0 \%$ carbohydrate, $22.0 \%$ protein) for 16 weeks. Lard was the major constituent of the HFD. Following acclimatization for 1 week, the rats were randomly divided into 2 experimental groups. One group included 8 rats that received the normal diet for 16 weeks (control group). The other group, including 24 animals, had ad libitum access to the HFD for 8 weeks, and after this period the rats were randomly divided into 3 groups. During the remaining 8 weeks, animals were provided the same ad libitum access to the HFD. In addition, one group was administered polydatin $30 \mathrm{mg} / \mathrm{kg} /$ day by gavage (8 rats, $\mathrm{HFD}+\mathrm{P} 30 \mathrm{mg} / \mathrm{kg}$ group), and another group was administered polydatin $90 \mathrm{mg} / \mathrm{kg} / \mathrm{day}$ by gavage (8 rats, $\mathrm{HFD}+\mathrm{P} 90 \mathrm{mg} / \mathrm{kg}$ group). The third group received the same amount of vehicle by gavage ( 8 rats, HFD group). Polydatin (3,4',5-trihydroxystilbene-3- $\beta$-mono-D-glucoside; purity, $>99 \%$ ) was obtained from Suzhou Baozetang Biotechnology Co., Ltd., Suzhou, China.

All rats were sacrificed after weighing at 16 weeks, and blood was drawn via the femoral artery and stored as plasma at $-80^{\circ} \mathrm{C}$. The intact liver was isolated and weighed. The liver index was calculated as the liver/body weight ratio. Sections from the right lobe were washed in cold saline and placed in 10\% formalin solution for histopathological analysis. The other samples were immediately frozen in liquid nitrogen and stored at $-80^{\circ} \mathrm{C}$ until use.

Histological analysis. The liver sections were paraffin-embedded, sliced into $5-\mu \mathrm{m}$ sections and stained with hematoxylin-eosin (HE) as previously described (15). The pathological changes were assessed and photographed under an Olympus BX-51 microscope. The liver biopsy was scored according to Brunt et al (16), as follows: 0, no steatosis; 1 , fatty hepatocytes occupying $<10 \%$ of the parenchyma; 2 , between $10 \%$ and $30 \% ; 3$, between $30 \%$ and $60 \%$; and 4 , fatty hepatocytes occupying $>60 \%$ of the parenchyma. Pathology was scored in a blinded manner by two independent pathologists with expertise in rodent liver.

Biochemical analysis. The activities of alanine aminotransferase (ALT) and aspartate aminotransferase (AST) in the plasma were measured using commercial enzyme assay kits (Wako Pure Chemical Industries, Osaka, Japan). Plasma TG, TC and free fatty acid (FFA) concentrations were determined using enzymatic reagent kits from Biosino (Beijing, China) according to the manufacturer's instructions. Hepatic lipids were extracted with chloroform-methanol (2:1) as described by Folch et al (17), and then dissolved using Triton X-100. TG, TC and FFA concentrations in the liver were determined using enzymatic reagent kits (Biosino) as previously described $(15,18)$.

ELISA assays for liver TNF- $\alpha$. For quantification of the liver TNF- $\alpha$ level, liver tissues were homogenized in extraction buffer $(50 \mathrm{mmol} / \mathrm{l}$ Tris, $150 \mathrm{mmol} / \mathrm{l} \mathrm{NaCl}, 1 \%$ Triton X-100 and a protease inhibitor cocktail). The homogenate was agitated on ice for $90 \mathrm{~min}$, centrifuged at $4^{\circ} \mathrm{C}$ for $15 \mathrm{~min}$, and then the precipitate was discarded. The TNF- $\alpha$ level was detected using a commercial enzyme-linked immunosorbent assay (ELISA) kit (BD Biosciences, Franklin Lakes, NJ, USA) according to the manufacturer's instructions.

Measurement of lipid peroxidation. Liver tissue (100 mg) was homogenized in 10 volumes of ice-cold Tris- $\mathrm{HCl}$ buffer containing $0.25 \mathrm{M}$ sucrose. The resulting homogenate was centrifuged at $3000 \mathrm{xg}$ for $10 \mathrm{~min}$ at $4^{\circ} \mathrm{C}$. The supernatant was used for assays of malondialdehyde (MDA)+4-hexanonenal (4-HNE). MDA+4-HNE was measured using a commercial enzyme assay kit (LPO-586) purchased from OXIS Health Products, Inc. (Portland, OR, USA).

Real-time RT-PCR analysis. Real-time PCR was used to detect changes in the expression of SREBP-1c, FAS, SCD1 and TNF- $\alpha$ mRNA in the liver tissues as previously described $(19,20)$. The isolation of total RNA from the liver tissues was performed using RNeasy Mini kits (Qiagen, Valencia, CA, USA) according to the manufacturer's instructions. RNA purity and concentration were determined spectrophotometrically. For each RT-PCR reaction, $2 \mu \mathrm{g}$ of total RNA was converted into cDNA using reverse transcriptase (Qiagen). Genomic DNA was eliminated using DNase I. For real-time PCR, the reactions were conducted by placing $10 \mu 1$ of material into 96-well plates with TaqMan PCR Master mix (Applied Biosystems Inc., Foster City, CA, USA). The specific primers and probes for SREBP-1c, FAS, SCD1 and TNF- $\alpha$ mRNA were obtained from Applied Biosystems, and RT-PCR was performed in an Applied Biosystems PRISM 7000 sequence detection system according to the manufacturer's instructions. A comparative cycle of threshold fluorescence (CT) method was used with $\beta$-actin as the internal control. The final results of real-time PCR were expressed as the ratio of the mRNA of interest to $\beta$-actin.

Statistical analysis. Results are expressed as the mean \pm SD. Statistical significance was evaluated with one-way ANOVA followed by Newman-Keuls test. $\mathrm{P}<0.05$ was considered to indicate a statistically significant difference.

\section{Results}

Effects of polydatin on liver index and plasma levels of ALT and AST in HFD rats. Following 16-week feeding, there was no difference in body weight gain among any group, but the HFD rats demonstrated a significantly higher liver index compared to the control rats. Polydatin ( 30 and $90 \mathrm{mg} / \mathrm{kg}$ ) treatment to HFD rats significantly reduced the liver index. The effects of polydatin on body weight, liver index and biochemical param- 
Table I. Effects of polydatin on body weight, liver index and biochemical parameters of the experimental rats.

\begin{tabular}{|c|c|c|c|c|}
\hline & Control & HFD & HFD+P 30 mg/kg & HFD+P 90mg/kg \\
\hline Body weight (g) & $482.31 \pm 31.52$ & $534.07 \pm 62.47$ & $507.64 \pm 52.13$ & $499.83 \pm 48.21$ \\
\hline Liver index & $3.11 \pm 0.37$ & $5.13 \pm 0.45^{\mathrm{b}}$ & $4.07 \pm 0.31^{\mathrm{b}, \mathrm{d}}$ & $3.62 \pm 0.29^{\mathrm{a}, \mathrm{d}}$ \\
\hline \multicolumn{5}{|l|}{ Plasma } \\
\hline ALT (U/l) & $51.02 \pm 4.13$ & $79.82 \pm 8.79^{b}$ & $62.17 \pm 5.32^{\mathrm{b}, \mathrm{d}}$ & $58.49 \pm 7.32^{\mathrm{d}}$ \\
\hline AST (U/l) & $63.72 \pm 5.41$ & $127.38 \pm 9.41^{\mathrm{b}}$ & $83.65 \pm 5.08^{\mathrm{b}, \mathrm{d}}$ & $75.42 \pm 4.93^{\mathrm{b}, \mathrm{d}}$ \\
\hline TG $(\mathrm{mmol} / \mathrm{l})$ & $0.68 \pm 0.13$ & $1.21 \pm 0.19^{b}$ & $0.87 \pm 0.31^{\mathrm{d}}$ & $0.96 \pm 0.35^{\mathrm{d}}$ \\
\hline $\mathrm{TC}(\mathrm{mmol} / \mathrm{l})$ & $1.73 \pm 0.27$ & $2.72 \pm 0.31^{\mathrm{b}}$ & $2.07 \pm 0.18^{\mathrm{a}, \mathrm{d}}$ & $2.16 \pm 0.19^{\mathrm{b}, \mathrm{d}}$ \\
\hline $\mathrm{HDL}(\mathrm{mmol} / \mathrm{l})$ & $1.42 \pm 0.13$ & $1.19 \pm 0.07^{\mathrm{b}}$ & $1.35 \pm 0.09^{\mathrm{d}}$ & $1.47 \pm 0.07^{\mathrm{d}}$ \\
\hline $\mathrm{LDL}(\mathrm{mmol} / \mathrm{l})$ & $0.22 \pm 0.02$ & $0.42 \pm 0.06^{\mathrm{b}}$ & $0.36 \pm 0.05^{\mathrm{b}, \mathrm{d}}$ & $0.33 \pm 0.07^{\mathrm{b}, \mathrm{d}}$ \\
\hline $\mathrm{FFA}(\mathrm{mmol} / \mathrm{l})$ & $0.86 \pm 0.08$ & $1.24 \pm 0.25^{\mathrm{b}}$ & $1.03 \pm 0.17^{\mathrm{d}}$ & $0.96 \pm 0.11^{\mathrm{d}}$ \\
\hline Insulin (mU/l) & $24.17 \pm 4.21$ & $30.25 \pm 5.83$ & $25.24 \pm 6.17$ & $22.35 \pm 6.73$ \\
\hline Glucose $(\mathrm{mmol} / \mathrm{l})$ & $6.23 \pm 0.65$ & $6.98 \pm 0.67$ & $6.42 \pm 0.66$ & $6.05 \pm 0.64$ \\
\hline \multicolumn{5}{|l|}{ Liver } \\
\hline $\mathrm{TG}(\mu \mathrm{mol} / \mathrm{g})$ & $4.37 \pm 0.23$ & $6.12 \pm 0.31^{\mathrm{b}}$ & $5.42 \pm 0.30^{\mathrm{b}, \mathrm{d}}$ & $4.76 \pm 0.35^{\mathrm{a}, \mathrm{d}}$ \\
\hline $\mathrm{TC}(\mu \mathrm{mol} / \mathrm{g})$ & $6.08 \pm 0.41$ & $7.69 \pm 0.37^{\mathrm{b}}$ & $7.13 \pm 032^{\mathrm{d}}$ & $6.46 \pm 0.36^{\mathrm{d}}$ \\
\hline $\mathrm{FFA}(\mu \mathrm{mol} / \mathrm{g})$ & $85.63 \pm 14.17$ & $157.9 \pm 24.70^{\mathrm{b}}$ & $139.11 \pm 16.9^{b, c}$ & $103.24 \pm 22.05^{\mathrm{d}}$ \\
\hline
\end{tabular}

Values are the mean $\pm \mathrm{SD}$ from 8 rats in each group. ${ }^{\mathrm{a}} \mathrm{P}<0.05$; ${ }^{\mathrm{b}} \mathrm{P}<0.01$ vs. control group; ${ }^{\mathrm{C}} \mathrm{P}<0.05 ;{ }^{\mathrm{d}} \mathrm{P}<0.01$ vs. HFD group. HFD, high-fat diet; HFD+P 30: high-fat diet plus polydatin $30 \mathrm{mg} / \mathrm{kg}$; HFD+P 90: high-fat diet plus polydatin $90 \mathrm{mg} / \mathrm{kg}$; ALT, alanine aminotransferase; AST, aspartate aminotransferase; TG, triglyceride; TC, total cholesterol; HDL, high-density lipoprotein; LDL, low-density lipoprotein; FFA, free fatty acids.
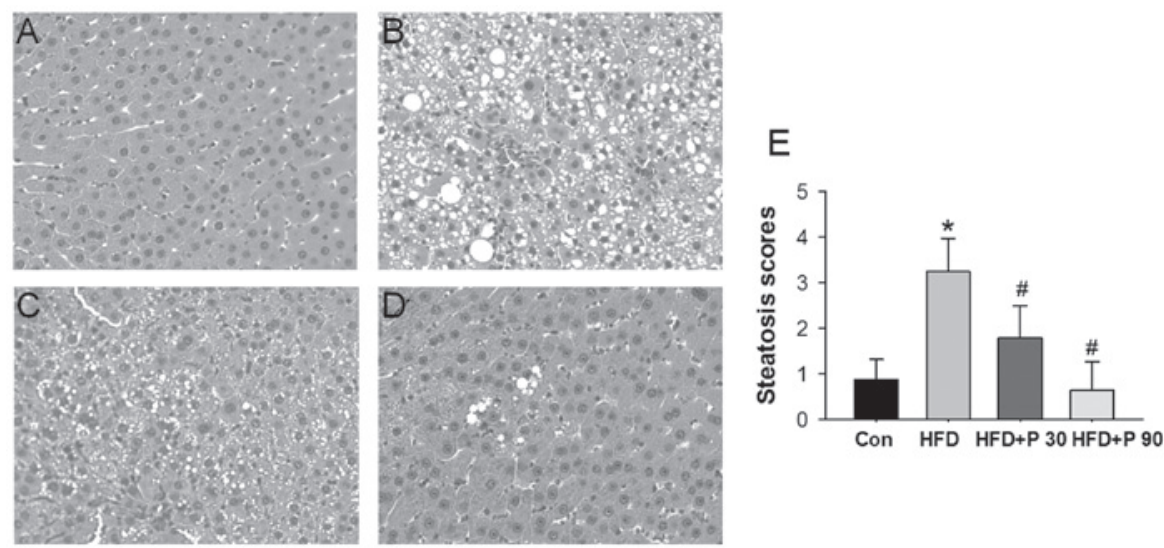

Figure 1. Effects of polydatin on liver histology in HFD rats. (A-D) Liver histologies of representative animals from each experimental group. Hematoxylin and eosin staining of liver sections at the end of the experiment of a representative rat from each experimental group, as described in Materials and methods. Original magnification, x40. (A) Standard chow diet (control group), (B) HFD group, (C) HFD plus polydatin 30 mg/kg (HFD+P 30), (D) HFD plus polydatin $90 \mathrm{mg} / \mathrm{kg}$ (HFD+P 90). (E) Liver histology scores. Steatosis was expressed as a score from 0 to 4 . ${ }^{*} \mathrm{P}<0.01 \mathrm{vs.} \mathrm{control;} \mathrm{"P<0.01} \mathrm{vs.} \mathrm{HFD.} \mathrm{Data} \mathrm{are} \mathrm{presented} \mathrm{as}$ the mean $\pm \mathrm{SD}$. Con, control group; HFD, high-fat diet.

eters of the experimental rats are shown in Table I. In addition, the biochemical analyses revealed increased plasma activities of ALT and AST in the HFD-fed rats compared to those of the control rats. HFD-induced elevations in plasma activities of ALT and AST were significantly reversed by treating the HFD rats with polydatin $(\mathrm{P}<0.05)$ (Table I).

Effects of polydatin on plasma and hepatic-lipid levels in HFD rats. To analyze the possible role of polydatin in lipid metabolism, a key factor associated with fatty liver formation, plasma and hepatic-lipid levels in the HFD rats were investigated.
The HFD-induced elevation in plasma concentrations of TG, TC, FFA and low-density lipoprotein (LDL) was significantly attenuated by polydatin, while decreased plasma high-density lipoprotein (HDL) levels were evidently reversed by treating the rats with polydatin (Table I). The hepatic accumulation of TC, TG, and FFA induced by the HFD was also significantly alleviated by treating the rats with polydatin. This suggests that polydatin is able to prevent hepatosteatosis via downregulation of the accumulation of lipids. These results indicate that polydatin had marked effects on the improvement of blood and hepatic lipid levels in the experimental rats. 

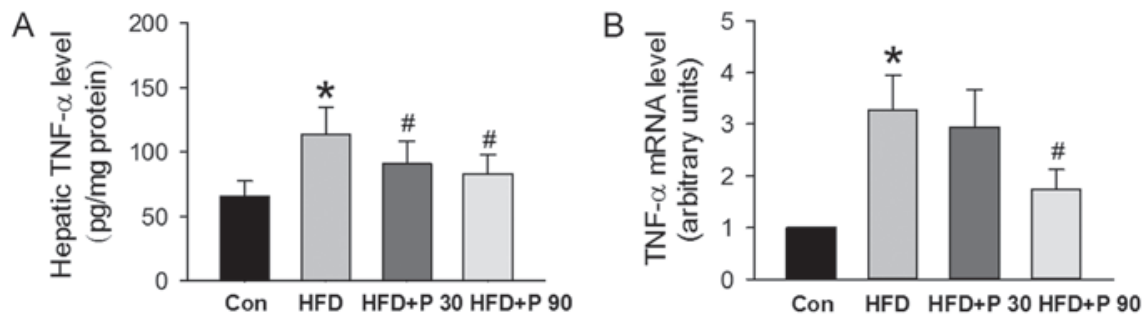

Figure 2. Effects of polydatin on hepatic TNF- $\alpha$ level in rats. (A) Hepatic TNF- $\alpha$ concentration was measured by ELISA analysis. (B) TNF- $\alpha$ mRNA expression in liver was examined by real-time RT-PCR analysis. Con, normal control group; HFD, high-fat diet; HFD+P 30, high-fat diet plus polydatin $30 \mathrm{mg} / \mathrm{kg}$; HFD+P 90: high-fat diet plus polydatin $90 \mathrm{mg} / \mathrm{kg}$. Data are presented as the mean $\pm \mathrm{SD}, \mathrm{n}=8$. " $\mathrm{P}<0.01 \mathrm{vs.} \mathrm{control;}{ }^{*} \mathrm{P}<0.01 \mathrm{vs}$. HFD. P, polydatin; TNF- $\alpha$, tumor necrosis factor- $\alpha$.

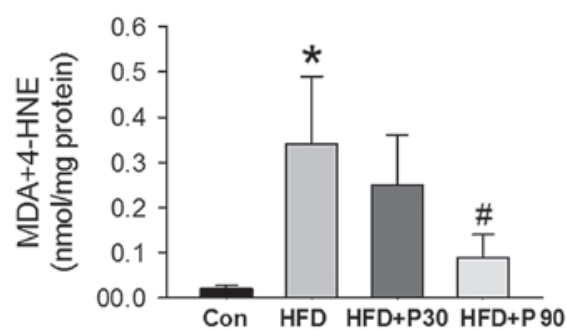

Figure 3. Effects of polydatin on lipid peroxide levels in the liver of HFD rats. Assays of MDA+4-HNE were conducted using a commercial enzyme assay kit. Con, normal control group; HFD, high-fat diet group; HFD+P 30, high-fat diet plus polydatin $30 \mathrm{mg} / \mathrm{kg} ; \mathrm{HFD}+\mathrm{P} 90$, high-fat diet plus polydatin $90 \mathrm{mg} / \mathrm{kg}$ Data are presented as the mean $\pm \mathrm{SD}, \mathrm{n}=8$. ${ }^{\mathrm{P}} \mathrm{P}<0.01$ vs. control; ${ }^{*} \mathrm{P}<0.01$ vs. HFD. P, polydatin. MDA, malondialdehyde; 4-HNE, 4-hexanonenal.

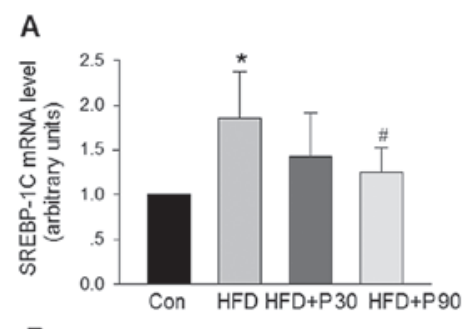

$\mathbf{B}$

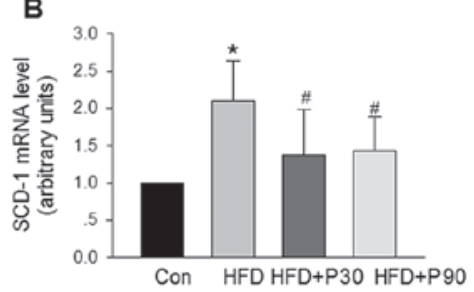

$c$

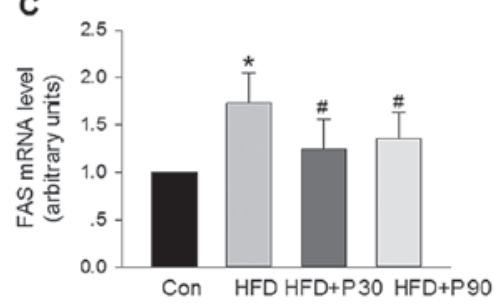

Figure 4. Effects of polydatin on hepatic mRNA expression of lipogenic genes in rats. Expression of hepatic SREBP-1c, FAS and SCD-1 in rats was examined by real-time RT-PCR analysis as described in Materials and methods. Con, normal control group; HFD, high-fat diet group; HFD+P 30, high-fat diet plus polydatin $30 \mathrm{mg} / \mathrm{kg}$; HFD $+\mathrm{P} 90$, high-fat diet plus polydatin $90 \mathrm{mg} / \mathrm{kg}$. Data are the mean $\pm \mathrm{SD}$ of the fold increase over control, $\mathrm{n}=8$. ${ }^{*} \mathrm{P}<0.05$ vs. control; ${ }^{*} \mathrm{P}<0.05$ vs. HFD. SREBP-1C, sterol-regulatory element binding protein; FAS, fatty acid synthase; SCD-1, stearoly-CoA desaturase 1.
Effects of polydatin on liver histology in HFD rats. Representative histological sections of the liver from each experimental group are shown in Fig. 1A-D. After 16 weeks, HFD-rats demonstrated severe hepatic microvesicular and macrovesicular fat. Rats treated with either 30 or $90 \mathrm{mg} / \mathrm{kg}$ polydatin significantly eliminated hepatic steatosis. The quantitative evaluation of the liver histology of all rats in each group is shown in Fig. 1E.

Effects of polydatin on hepatic TNF- $\alpha$ level. Hepatic steatosis is associated with increased hepatic TNF- $\alpha$, which stimulates lipogenesis as well as induces hepatic dysfunction. ELISA analysis demonstrated that hepatic TNF- $\alpha$ protein was greater in HFD rats and lower in HFD rats treated with polydatin at 30 or $90 \mathrm{mg} / \mathrm{kg}$ (Fig. 2A). In addition, hepatic TNF- $\alpha$ mRNA expression was 3 -fold greater in HFD rats than in normal control rats. Polydatin $(90 \mathrm{mg} / \mathrm{kg})$ administration, however, caused a significant decrease in TNF- $\alpha$ mRNA expression (Fig. 2B).

Effects of polydatin on lipid peroxidation in liver homogenates. To investigate whether polydatin is capable of preventing lipid peroxidation, we measured the formation of MDA and 4-HNE in liver tissues. The levels of MDA and 4-HNE were significantly higher in the HFD rats compared to the control rats. Supplementation of polydatin $90 \mathrm{mg} / \mathrm{kg}$ resulted in a reduction of MDA and 4-HNE levels ( $\mathrm{P}<0.01$ vs. HFD rats (Fig. 3).

Effects of polydatin on relative $m R N A$ concentrations of SREBP-lc and its target genes in the liver of HFD rats. We examined the expression of genes regulating lipogenesis to define how polydatin inhibits hepatic steatosis. In the HFD rats, the expression of SREBP-1c, FAS and SCD-1 was significantly upregulated in the liver (Fig. 4). Polydatin at $90 \mathrm{mg} / \mathrm{kg}$ decreased the expression of liver SREBP-1c mRNA; while the two doses of polydatin decreased liver FAS and SCD-1 mRNA levels significantly (Fig. 4).

\section{Discussion}

In the present study, the results demonstrated that polydatin was able to decrease the TC, TG and FFA levels in serum and liver tissue, as well as the hepatic weight index in high-fat diet-induced fatty liver. The histopathological evaluation of liver specimens demonstrated that polydatin may decrease lipid accumulation, particularly in $90 \mathrm{mg} / \mathrm{kg}$ polydatin groups, and the hepatic lipid accumulation in a few rats was reversed. 
These results suggested that polydatin may exert a therapeutic effect on high-fat diet-induced fatty liver in rats.

TNF- $\alpha$ is a cytokine which plays a central role in the chronic inflammatory response in fatty liver $(21,22)$. Kupffer cells are the major source of increased TNF- $\alpha$ secretion within the liver (21). TNF- $\alpha$ mRNA is increased in liver and adipose tissues in NASH patients (23-24). TNF receptor knockout mice have less severe steatosis than wild-type littermates, and a significant reduction in liver injury in steatohepatitis following anti-TNF- $\alpha$ therapy supports the pathogenic role of TNF- $\alpha$ (25-27). In this study, TNF- $\alpha$ mRNA and protein was higher in the HFD group, but was attenuated by polydatin treatment. One of the mechanisms for lowering TNF- $\alpha$ levels may have been the anti-inflammatory properties of polydatin. Previous studies have indicated that polydatin from Polygonum cuspidatum may regulate the inflammatory response and immune function $(10,12)$. For example, polydatin was identified to regulate IL-17 production in human peripheral blood mononuclear cells and nuclear factor $\kappa \mathrm{B}$ activation in the rat model of cerebral artery occlusion (12,28-29). Another mechanism for regulating the inflammatory response may be the decrease in liver fat and oxidative stress, which indirectly decreases the damage of hepatocytes and lowers the TNF- $\alpha$ level. In this study, the increase in MDA and 4-HNE, a marker of oxidative stress, was significantly attenuated by polydatin. These results suggest that polydatin may inhibit the inflammatory response in NASH and further decrease damage to the liver.

In this study, we also examined the effects of polydatin on hepatic SREBP-1c mRNA expression in rats. The results indicated that the expression of SREBP-1c mRNA in the HFD group was higher than that in the control group. Following administration of polydatin $30-90 \mathrm{mg} / \mathrm{kg}$ for 8 weeks, SREBP-1c mRNA expression was decreased. It was suggested that inhibition of SREBP-1c by polydatin may counteract the high-fat diet-induced fatty liver via regulation of SREBP-1c-mediated target gene expression, and subsequently correct the imbalance of lipid metabolism toward lipid accumulation in the level of genetic transcription.

SREBP-1c is a member of the family of SREBP membranebound transcription factors. It mainly activates the transcription of lipogenic genes that contain sterol regulatory elements in their promoter regions, including FAS and SCD-1 (30,31). FAS is a key enzyme which controls the rate of fatty acid synthesis. Increase in the expression of FAS may increase fatty acid synthesis and lead to the ectopic overaccumulation of fatty acids and TGs in the liver (32). Several lines of evidence have suggested that the induction of SREBP-1c gene expression in the liver contributes to the elevation of hepatic TG concentrations, leading to the development of NAFLD (31-37). As supporting evidence, SREBP-1c overexpression in transgenic mice leads to fatty liver, while inactivation of the SREBP-1c gene in the livers of ob/ob mice reduces hepatic TG content by $50 \%(33,34)$. Shrestha et al demonstrated that dietary catechins extracted from green tea extract could reduce plasma and hepatic TG concentration in fructose-fed rats, mainly through downregulation of SREBP-1c and its target gene (37). Notably, we identified that SREBP-1c mRNA expression was stimulated by HFD, and consumption of polydatin suppressed its expression. As a result of low SREBP-1c expression in rats fed polydatin, there was a concomitant significant reduction in the expression of FAS and SCD-1 mRNA. The results suggested that the stimulated FAS and SCD-1 transcription may occur following SREBP-1c activation caused by HFD. Polydatin is likely to have an inhibitory effect on SREBP-1c expression, which in turn influences transcription in these lipogenic genes, thereby reducing enzyme activity and resulting in a low rate of lipid synthesis.

Recent evidence indicates that key liver genes involved in TG homeostasis are regulated by insulin (38-42). However, significant differences in the plasma concentrations of insulin and glucose were not observed in our study. The failure of HFD to affect plasma insulin concentration in this study could be due to the fact that we focused on a single measurement of hormone concentration at one point in time. The measurement of plasma insulin concentration in defined time intervals following the serving of food according to a fixed time schedule, combined with the determination of the area under the curve of insulin, should be elucidated in further studies.

In conclusion, this study demonstrates that polydatin may improve the liver function of rats with non-alcoholic steatohepatitis by lowering lipid levels in the blood and liver, reducing oxidative damage and inflammation, and regulating the gene expression of hepatic fatty acid biosynthesis. These polydatin effects appear to be dose-dependent and are likely to be mediated via alterations in adipose lipid metabolism and improvements in hepatic anti-inflammatory responses and antioxidant defenses. Further studies are necessary to clarify the mechanisms behind the beneficial effects of polydatin on NAFLD.

\section{Acknowledgements}

This study was supported by the National Natural Science Foundation of China (No. 31171101 and No. 81100175) and the Shaanxi Provincial Department of Education (No. 08JK274).

\section{References}

1. Duvnjak M, Lerotić I, Barsić N, Tomasić V, Virović Jukić L and Velagić V: Pathogenesis and management issues for non-alcoholic fatty liver disease. World J Gastroenterol 13: 4539-4550, 2007.

2. Angulo P: Non-alcoholic fatty liver disease. N Engl J Med 346: 1221-1231, 2002.

3. Yeh MM and Brunt EM: Pathology of non-alcoholic fatty liver disease. Am J Clin Pathol 128: 837-847, 2007.

4. Farrell GC and Larter CZ: Non-alcoholic fatty liver disease: from steatosis to cirrhosis. Hepatology 43: S99-S112, 2006.

5. Postic C and Girard J: The role of the lipogenic pathway in the development of hepatic steatosis. Diabetes Metab 34: 643-648, 2008.

6. Ayyad C and Andersen T: Long-term efficacy of dietary treatment of obesity: a systematic review of studies published between 1931 and 1999. Obes Rev 1: 113-119, 2000.

7. Xue L: Progress in the pharmacological study of Chinese herbal drug: Polygonum cuspidatum. Zhongguo Zhong Yao Za Zhi 25: 651-653, 2000.

8. Luper S: A review of plants used in the treatment of liver disease: part two. Altern Med Rev 4: 178-188, 1999.

9. Kimura Y: Pharmacological studies on resveratrol. Methods Find Exp Clin Pharmacol 25: 297-310, 2003.

10. Zhang H, Dou C and Gu F: Advances in the study on pharmacological actions of Polygonum cuspidatum Sieb: clearing heat and detoxication. Zhong Yao Cai 26: 606-610, 2003.

11. Huang ZS, Wang ZW, Liu MP, Zhong SQ, Li QM and Rong XL: Protective effects of polydatin against $\mathrm{CCl}(4)$-induced injury to primarily cultured rat hepatocytes. World J Gastroenterol 5: 41-44, 1999. 
12. Ji H, Zhang X, Du Y, Liu H, Li S and Li L: Polydatin modulates inflammation by decreasing NF- $\kappa \mathrm{B}$ activation and oxidative stress by increasing Gli1, Ptch1, SOD1 expression and ameliorates blood-brain barrier permeability for its neuroprotective effect in pMCAO rat brain. Brain Res Bull 87: 50-59, 2012.

13. Du J, Sun LN, Xing WW, et al: Lipid-lowering effects of polydatin from Polygonum cuspidatum in hyperlipidemic hamsters. Phytomedicine 16: 652-658, 2009.

14. Xing WW, Wu JZ, Jia M, Du J, Zhang H and Qin LP: Effects of polydatin from Polygonum cuspidatum on lipid profile in hyperlipidemic rabbits. Biomed Pharmacother 63: 457-462, 2009.

15. Zhang Q, Zhao Y, Zhang DB and Sun LJ: Effect of Sinai san decoction on the development of non-alcoholic steatohepatitis in rats. World J Gastroenterol 11: 1392-1395, 2005.

16. Brunt EM, Janney CG, Di Bisceglie AM, Neuschwander-Tetri BA and Bacon BR: Nonalcoholic steatohepatitis: a proposal for grading and staging the histological lesions. Am J Gastroenterol 94: 2467-2474, 1999.

17. Folch J, Lees M, Sloane and Starley GH: A simple method for the isolation and purification of total lipids from animal tissues. J Biol Chem 226: 497-509, 1957.

18. Bartlett GR: Phosphorous assay in column chromatography. J Biol Chem 234: 466-468, 1959.

19. Zhang Q and Tan Y: Nerve growth factor augments neuronal responsiveness to noradrenaline in cultured dorsal root ganglion neurons of rats. Neuroscience 193: 72-79, 2011

20. Zhang Q, Yao F, Raizada MK, O'Rourke ST and Sun C: Apelin gene transfer into the rostral ventrolateral medulla induces chronic blood pressure elevation in normotensive rats. Circ Res 104: 1421-1428, 2009.

21. Diehl AM, Li ZP, Lin HZ and Yang SQ: Cytokines and the pathogenesis of non-alcoholic steatohepatitis. Gut 54: 303-306, 2005.

22. Tilg H and Diehl AM: Cytokines in alcoholic and nonalcoholic steatohepatitis. N Engl J Med 343: 1467-1476, 2000.

23. Crespo J, Cayon A, Fernandez-Gil P, et al: Gene expression of tumor necrosis factor alpha and TNF-receptors, p 55 and p75, in nonalcoholic steatohepatitis patients. Hepatology 34: 1158-1163, 2001.

24. Abiru S, Migita K, Maeda Y, et al: Serum cytokine and soluble cytokine receptor levels in patients with non-alcoholic steatohepatitis. Liver Int 26: 39-45, 2006.

25. Poniachik J, Csendes A, Diaz JC, et al: Increased production of IL-1alpha and TNFalpha in lipopolysaccharide-stimulated blood from obese patients with non-alcoholic fatty liver disease. Cytokine 33: 252-257, 2006.

26. Tomita K, Tamiya G, Ando S, et al: Tumour necrosis factor alpha signaling through activation of Kupffer cells plays an essential role in liver fibrosis of non-alcoholic steatohepatitis in mice. Gut 55: 415-424, 2006.

27. Hui JM, Hodge A, Farrell GC, Kench JG, Kriketos A and George J: Beyond insulin resistance in NASH: TNF-alpha or adiponectin. Hepatology 40: 46-54, 2004.
28. Lanzilli G, Cottarelli A, Nicotera G, Guida S, Ravagnan G and Fuggetta MP: Anti-inflammatory effect of resveratrol and polydatin by in vitro IL-17 modulation. Inflammation 35: 240-248, 2012

29. Wang X, Song R, Bian HN, Brunk UT, Zhao M and Zhao KS Polydatin protects arterial smooth muscle cells against mitochondrial dysfunction and lysosomal destabilization following hemorrhagic shock. Am J Physiol Regul Integr Comp Physiol: Jan 25, 2002 (Epub ahead of print).

30. Shimano H: Sterol regulatory element-binding proteins (SREBPs): transcriptional regulators of lipid synthetic genes Prog Lipid Res 40: 439-452, 2001.

31. Raghow R, Yellaturu C, Deng X, Park EA and Elam MB: SREBPs: the crossroads of physiological and pathological lipid homeostasis. Trends Endocrinol Metab 19: 65-73, 2008.

32. Yan D, Lehto M, Rasilainen L, et al: Oxysterol binding protein induces upregulation of SREBP-1c and enhances hepatic lipogenesis. Arterioscler Thromb Vasc Biol 27: 1108-1114, 2007.

33. Yahagi N, Shimano H, Hasty AH, Matsuzaka T, Ide T and Yoshikawa T: Absence of sterol regulatory element-binding protein-1 (SREBP-1) ameliorates fatty livers but not obesity or insulin resistance in Lep(ob)/Lep(ob) mice. J Biol Chem 277: 19353-19357, 2002.

34. Shimomura I, Bashmakov Y and Horton JD: Increased levels of nuclear SREBP-1c associated with fatty livers in two mouse models of diabetes mellitus. J Biol Chem 274: 30028-30032, 1999.

35. Ahmed MH and Byrne CD: Modulation of sterol regulatory element binding proteins (SREBPs) as potential treatments for non-alcoholic fatty liver disease (NAFLD). Drug Discov Today 12: 740-747, 2007.

36. Ntambi JM, Miyazaki M, Stoehr JP, et al: Loss of stearoyl-CoA desaturase-1 function protects mice against adiposity. Proc Natl Acad Sci USA 99: 11482-11486, 2002.

37. Shrestha S, Ehlers SJ, Lee JY,Fernandez ML and Koo SI: Dietary green tea extract lowers plasma and hepatic triglycerides and decreases the expression of sterol regulatory element-binding protein-1c mRNA and its responsive genes in fructose-fed, ovariectomized rats. J Nutr 139: 640-645, 2009.

38. Saltiel A and Kahn CR: Insulin signalling and the regulation of glucose and lipid metabolism. Nature 414: 799-806, 2001.

39. Browning $J$ and Horton J: Molecular mediators of hepatic steatosis and liver injury. J Clin Invest 114: 147-152, 2004

40. Utzschneider KM and Kahn SE: Review: the role of insulin resistance in nonalcoholic fatty liver disease. J Clin Endocrinol Metab 91: 4753-4761, 2006.

41. Previs SF, Withers DJ, Ren JM, White MF and Shulman GI: Contrasting effects of IRS-1 versus IRS-2 gene disruption on carbohydrate and lipid metabolism in vivo. J Biol Chem 275: 38990-38994, 2000

42. Samuel VT, Liu ZX, Qu X, et al: Mechanism of hepatic insulin resistance in non-alcoholic fatty liver disease. J Biol Chem 279: 32345-32353, 2004. 